\title{
VALIDITY OF THE C-RDS SELF-REPORTED RISKY DRIVING MEASURE
}

\author{
Bruce G. Simons-Morton ${ }^{1}$, Kaigang Li $^{1}$, Ashley Brooks-Russell ${ }^{1}$, Johnathon Ehsani ${ }^{1}$, Anuj Pradhan ${ }^{1}$, \\ Marie Claude Ouimet ${ }^{2}$ \& Sheila Klauer ${ }^{3}$ \\ ${ }^{1}$ National Institutes of Health, Bethesda, Maryland, USA \\ ${ }^{2}$ University of Sherbrooke, Montreal, Quebec, Canada \\ ${ }^{3}$ Virginia Tech Transportation Institute, Virginia Polytechnic Institute and State University, \\ Blacksburg, Virginia, USA \\ Email: mortonb@mail.nih.gov
}

\begin{abstract}
Summary: This study examined the reliability and validity of the Checkpoints Risky Driving Scale (C-RDS) in relation to the Dula Dangerous Driving Index (DDDI) and an objective measure of risky driving. Naturalistic and survey data were collected over an 18-month period from 42 newly-licensed teenage drivers. Kinematic Risky Driving was operationally defined as the rate of elevated gravitational-force events per 100 miles obtained from accelerometers and global positioning systems. Two self-report measures of risky driving, the C-RDS and the DDDI, were assessed at 6-months, 12-months, and 18-months after licensure. Reliability was examined for each measure with correlations and autoregressive models over three time points. Validity was assessed by correlations between the measures and cross-lagged autoregressive models of the longitudinal association of self-reported measures with Kinematic Risky Driving and vice versa. Both the C-RDS and DDDI measures demonstrated substantial stability over time and were highly correlated with each other. The C-RDS measure was significantly associated with Kinematic Risky Driving. The findings provide evidence for the reliability and validity of C-RDS.
\end{abstract}

\section{INTRODUCTION}

Risky driving is implicated in crashes, particularly among young drivers. Analyses of the Fatal Accident Reporting database indicate that teenagers have extremely high fatal crash rates relative to experienced adults (Ouimet et al., 2010). Teen crashes tend to involve high speeds, late night, and multiple teenage passengers (Williams, 2003). Teenagers drive faster and allow shorter headway than older drivers (Simons-Morton et al., 2006; McKenna et al., 1998) and make many errors (McKnight \& McKnight, 2003).

Generally, population prevalence estimates of risky driving employ self-reported measures of risky driving (McCartt, Shabanova, \& Leaf, 2003; Mayhew et al., 2003), although prevalence of risky driving can be inferred from crash analyses datasets (Williams, 2003; Braitman, et al., 2008; Curry et al., 2011). The reliability and validity of self-report measures have relied on other self-reported measures such as traffic violations, reported motor vehicle crash involvement, and archival police-reported driver history records (Shope \& Bingham, 2002; Dula \& Ballard, 2003; Willemsen et al., 2008). No studies have compared self-report measures with objective measures of risky driving using instrumented vehicle data, presumably because there have been few naturalistic driving studies due to their complexity and expense. 
The Naturalistic Teen Driving Study (NTDS) provided a unique opportunity to assess the reliability and validity of C-RDS with objective measures of risky driving. The purpose of this paper is to report the relationships over time between the C-RDS and a widely used self-reported measure of risky driving, Dula Dangerous Driving Index (DDDI), and an objective measure of risky driving.

\section{METHODS}

A data acquisition system was installed in the vehicles of 42 newly-licensed teenage drivers 16 years old during their first 18 months of independent driving. It consisted of cameras, accelerometers, Global Positioning System (GPS), various other sensors, and a computer with removable hard drive. Questionnaires administered at 6-months, 12-months, and 18-months included the C-RDS and DDDI self-report measures.

\section{Measures}

Kinematic Risky Driving. Accelerometers continuously assessed gravitational-force (g-force) events and GPS data enabled the calculation of events per mile/kilometer. Kinematic Risky Driving was defined by the combined rates per 100 miles of g-force events $\left(1 \mathrm{~g}=9.8 \mathrm{~m} / \mathrm{s}^{2}\right)$ at the following thresholds: longitudinal deceleration/hard braking $(\leq-0.45 \mathrm{~g})$, longitudinal acceleration/rapid starts $(\geq 0.35 \mathrm{~g})$, lateral negative/hard left turn $(\leq-0.50 \mathrm{~g})$ and lateral positive/hard right turn accelerations ( $\geq 0.50 \mathrm{~g}$ ), and yaw ( \pm 6 degrees within 3 seconds). The individual items were associated with the composite measure (hereafter referred to as Kinematic Risky Driving), with correlations ranging from $r=0.60$ to 0.84 , providing reasonable internal consistency, [standardized Cronbach's $\alpha$ (hereafter referred to as $\alpha$ ) $=0.78$ ]. Kinematic Risky Driving was correlated with crash/near crash rates $(r=0.60 ; p<0.001)$. Previous analyses indicated that higher Kinematic Risky Driving in the previous month predicted the likelihood of a crash or near crash in the following month and an area under the curve of 0.74, indicating high measurement sensitivity (Simons-Morton et al., 2012). Kinematic Risky Driving was nearly 4 times higher for novice teenagers than adults over the first 18 months of teen licensure (SimonsMorton et al., 2011). Kinematic Risky Driving was aggregated over 18 months and also over 6month periods to correspond with the self-report assessments at 6-months (T1), 12-months (T2), and 18-months (T3).

Checkpoints Risky Driving Scale (C-RDS). The C-RDS, employed in a number of studies (Hartos et al., 2002; Simons-Morton et al., 2006), consists of 19 items that ask about the prevalence of speeding, aggressive driving, and other risky driving behaviors. A list of items is provided in Table 1.

Table 1. Checkpoints Risky Driving Scale Questionnaire

\begin{tabular}{|llrl|l|}
\hline \multicolumn{3}{|l|}{ In the last 7 days, how often did you do each of the following (when you had the occasion to): } \\
\hline 1 & $\begin{array}{l}\text { Exceeded the speed limit in residential or school } \\
\text { zones }\end{array}$ & 11 & $\begin{array}{l}\text { Went through an intersection when the light was red } \\
\text { or just turning red }\end{array}$ \\
2 & $\begin{array}{l}\text { Drove } 10-19 \text { miles per hour over the speed limit } \\
3\end{array}$ & $\begin{array}{l}\text { Drove } 20 \text { or more miles per hour over the speed } \\
\text { limit }\end{array}$ & 13 & Went through a stop sign without stopping completely \\
4 & Purposely tailgated or followed another vehicle very & 14 & Changed lanes without signaling \\
\hline
\end{tabular}




\begin{tabular}{|c|c|c|c|}
\hline & closely & & \\
\hline 5 & Switched lanes to weave through slower traffic & 15 & $\begin{array}{l}\text { Played the radio so loudly you wouldn't be able to } \\
\text { hear other vehicle horns or sirens }\end{array}$ \\
\hline 6 & $\begin{array}{l}\text { Changed lanes with very little room between } \\
\text { vehicles }\end{array}$ & 16 & Drove without wearing a seat belt \\
\hline 7 & Cut in front of a vehicle to turn & 17 & Raced another vehicle, even just for a short distance \\
\hline 8 & $\begin{array}{l}\text { Pulled out into traffic without waiting for a large } \\
\text { space between vehicles }\end{array}$ & 18 & $\begin{array}{l}\text { Read, ate, talked on a cell phone, put on makeup, } \\
\text { horsed around with passengers, or other such } \\
\text { activities while driving }\end{array}$ \\
\hline 9 & Made an illegal U-turn & 19 & Drove in a way to show off to other people \\
\hline 10 & $\begin{array}{l}\text { Went through an intersection when the light was } \\
\text { yellow or just turning yellow }\end{array}$ & & \\
\hline
\end{tabular}

The scale is: NA (0 - coded to missing when analyzing the data); Never (1); Rarely (2); Some-times (3);

Often (4); and Always (5).

DULA Dangerous Driving Index (DDDI). This 28-item measure was designed to assess the frequency (5 point scale from never to always) of deliberately aggressive (7 items), risky (12 items; constituting the risky driving subscale), and negative-emotion (9 items) driving that endangers the safety of the driver and other road users (Dula \& Ballard, 2003). In previous research the measures demonstrated good internal consistency $(\alpha=0.83-0.93)$ and temporal stability ( $r=0.55-0.73)$ (Dula \& Ballard, 2003; Willemsen et al., 2008). Willemsen and colleagues (2008) reported that both the total score and the subscales discriminate between traffic offenders and non-offenders. Richer and Bergeron (2012) reported significant associations between the DDDI and several criterion measures, including traffic fines, self-reported collisions, and maximum speed in simulated driving. Both the DDDI and the DDDI risky driving subscale were included in this study. The total DDDI score ( $\alpha=.91$, .91, and .86 at T1, T2, and T3) and DDDI risky driving subscale (12 items; $\alpha=.86, .83$, and .76 at T1, T2, and T3) were found to have good internal consistency at all three time points.

\section{Analyses}

Intra-class correlations (Cronbach's alpha coefficients) were calculated to assess internal consistency of the self-report scales at each time point and the 18-month Kinematic Risky Driving measure.

Pearson correlations were calculated between C-RDS at each time point (T1, T2, and T3) and the corresponding measures of DDDI and Kinematic Risky Driving as well as with the 18 month measure of Kinematic Risky Driving. Auto-regressive cross-lagged models were created to assess temporal stability of C-RDS, DDDI and Kinematic Risky Driving across the three time points and relationships between C-RDS and DDDI with Kinematic Risky Driving from T1 to $\mathrm{T} 2$ and from T2 to T3. Auto-regressive models are a form of time series analysis that accounts for the linear correlation of variables measured at multiple time points. The betas provided by the cross-lagged comparisons are interpreted in the same manner as in all regression analyses. Statistical analyses were performed using SAS 9.2 and Mplus 7. 


\section{RESULTS}

$C$-RDS and DDDI. Good internal consistency of the C-RDS was demonstrated at all three time points (alphas at T1, T2, and T3 $=0.90,0.90$, and 0.89 ). As shown in the correlation matrix in Table 2, C-RDS at each time point (T1, T2, and T3) was significantly associated with DDDI and DDDI Risky Driving Subscale measured at the same time points (T1, T2, and T3).

C-RDS and Kinematic Risky Driving. As shown in Table 2, C-RDS at T1, T2, and T3 was significantly associated with overall 18-month Kinematic Risky Driving, and C-RDS at T2 and T3 was significantly associated with Kinematic Risky Driving at T2 and T3. In auto-regressive cross-lagged analysis (Figure 1), Kinematic Risky Driving at T1 was significantly associated with Kinematic Risky Driving at T2, and Kinematic Risky Driving at T2 was significantly associated with Kinematic Risky Driving at T3. Similarly, C-RDS at T1 was significantly associated with C-RDS at T2, and C-RDS at T2 was significantly associated with C-RDS at T3. However, no significant cross-lagged associations between C-RDS and Kinematic Risky Driving were found.

DDDI and Kinematic Risky Driving. As shown in Table 2, only the T1 assessment of the DDDI was associated with 18-month Kinematic Risky Driving. The DDDI risky driving subscale measure at T1, T2, and T3 was significantly associated with overall 18-month Kinematic Risky Driving, and the measure at T2 and T3 was significantly associated with Kinematic Risky Driving at T2 and T3, all at higher levels than the correlations between DDDI and Kinematic Risky Driving. As shown in Figure 2, in auto-regressive cross-lagged analyses DDDI at T1 was significantly associated with DDDI at T2 and DDDI at T2 was significantly associated with DDDI at T3. However, no significant cross-lagged associations between DDDI and Kinematic Risky Driving were found.

Table 2. Correlation between Kinematic Risky Driving and self-reported measures of risky driving

\begin{tabular}{|c|c|c|c|c|c|c|c|c|c|c|c|c|c|c|}
\hline & 1 & & 2 & 3 & 4 & 5 & 6 & 7 & 8 & 9 & 10 & 11 & 12 & 13 \\
\hline 1 & 18m KRD & 1 & & & & & & & & & & & & \\
\hline 2 & T1 KRD & $0.87^{* * *}$ & 1 & & & & & & & & & & & \\
\hline 3 & T2 KRD & $0.96^{* * *}$ & $0.77^{* * *}$ & 1 & & & & & & & & & & \\
\hline 4 & T3 KRD & $0.93^{* * *}$ & $0.70^{* * *}$ & $0.89^{* * *}$ & 1 & & & & & & & & & \\
\hline 5 & T1 DDDI & $0.36^{*}$ & 0.21 & $0.31^{*}$ & $0.37^{*}$ & 1 & & & & & & & & \\
\hline 6 & T2 DDDI & 0.27 & 0.14 & 0.28 & 0.29 & $0.87^{* * *}$ & 1 & & & & & & & \\
\hline 7 & T3 DDDI & 0.25 & 0.13 & 0.28 & 0.26 & $0.81^{* * *}$ & $0.85^{* * *}$ & 1 & & & & & & \\
\hline 8 & T1 DDDI RD & $0.40^{* *}$ & 0.20 & $0.40^{* *}$ & $0.42^{* *}$ & $0.83^{* * *}$ & $0.71^{* * *}$ & $0.60^{* * *}$ & 1 & & & & & \\
\hline 9 & T2 DDDI RD & $0.50^{* * *}$ & 0.28 & $0.54^{* * *}$ & $0.51^{* * *}$ & $0.77^{* * *}$ & $0.82^{* * *}$ & $0.60^{* * *}$ & $0.86^{* * *}$ & 1 & & & & \\
\hline 10 & T3 DDDI RD & $0.49^{* * *}$ & 0.26 & $0.55^{* * *}$ & $0.49^{* * *}$ & $0.78^{* * *}$ & $0.78^{* * *}$ & $0.78^{* * *}$ & $0.80^{* * *}$ & $0.86^{* * *}$ & 1 & & & \\
\hline 11 & T1 C-RDS & $0.44^{* * *}$ & 0.28 & $0.41^{* *}$ & $0.46^{* * *}$ & $0.74^{* * *}$ & $0.69^{* * *}$ & $0.67^{* * *}$ & $0.74^{* * *}$ & $0.77^{* * *}$ & $0.78^{* * *}$ & 1 & & \\
\hline 12 & T2 C-RDS & $0.41^{* *}$ & 0.25 & $0.45^{* * *}$ & $0.40^{* *}$ & $0.62^{* * *}$ & $0.77^{* * *}$ & $0.67^{* * *}$ & $0.64^{* * *}$ & $0.80^{* * *}$ & $0.81^{* * *}$ & $0.83^{* * *}$ & 1 & \\
\hline 13 & T3 C-RDS & $0.35^{*}$ & 0.23 & $0.40^{* *}$ & $0.35^{*}$ & $0.70^{* * *}$ & $0.78^{* * *}$ & $0.85^{* * *}$ & $0.52^{* * *}$ & $0.60^{* * *}$ & $0.75^{* * *}$ & $0.73^{* * *}$ & $0.80^{* * *}$ & 1 \\
\hline
\end{tabular}

Notes: $18 \mathrm{~m}$ KRD= 18 month Kinematic Risky Driving (g-force events rate); $\mathrm{T} 1$ = 6 months; $\mathrm{T} 2=12$ months; $\mathrm{T} 3=18$ months; DDDI = DULA Dangerous Driving Index; DDDI RD = DDDI risky driving subscale; C-RDS = Checkpoints Risky Driving Scale. 


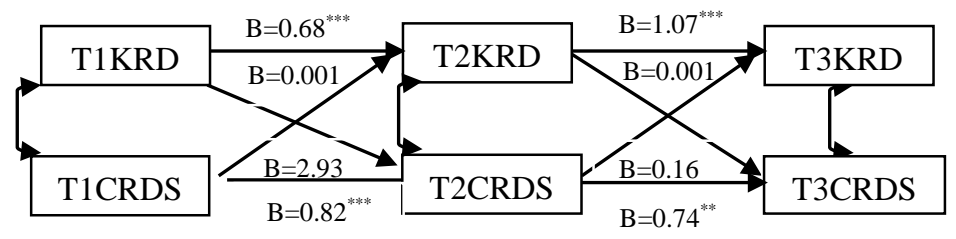

Figure 1. Results of cross-lagged model (Kinematic Risky Driving vs. Checkpoints Risky Driving

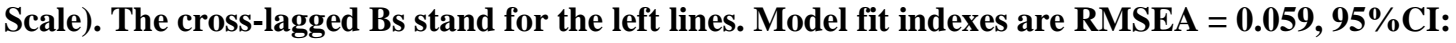
$<.001,0.247, \mathrm{AIC}=836.7, \mathrm{BIC}=876.7$, and adjusted $\mathrm{BIC}=\mathbf{8 0 4 . 7}$

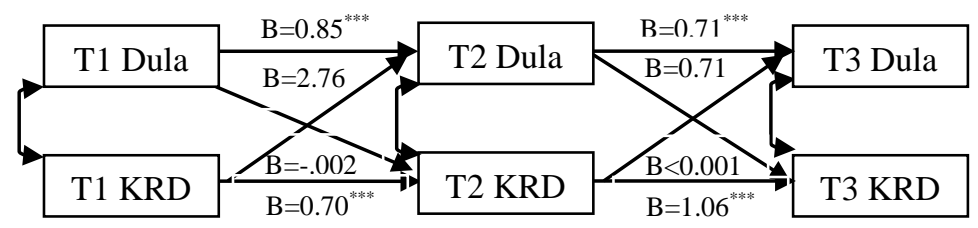

Figure 2. Results of cross-lagged model (Dula Dangerous Driving Index vs. Kinematic Risky Driving). The cross-lagged Bs stand for the left lines. Model fit indexes are RMSEA $=0.167,95 \% \mathrm{CI}$ : $<.001,0.321, \mathrm{AIC}=755.2, \mathrm{BIC}=795.1$, and adjusted $\mathrm{BIC}=723.1$

\section{DISCUSSION}

C-RDS was highly correlated over time with the DDDI and the DDDI subscale on risky driving. C-RDS was also highly correlated with the T2, T3, and 18-month Kinematic Risky Driving measures. The DDDI Risky Driving Subscale was associated with Kinematic Risky Driving at similar levels to the C-RDS and at higher levels and more consistently than the DDDI.

The advantages of self-report measures of risky driving include their low cost and ease of administration, which allows researchers to collect data on large samples to assess prevalence and individual variability. Despite their popularity, surprisingly little research has been conducted on the reliability and validity of existing measures of self-reported risky driving. The DDDI is perhaps the best studied self-report measure and is the only measure that has been evaluated cross-culturally (Willemsen et al., 2008). While the full DDDI has often been used, its interpretation is uncertain because it includes aggressive, risky, and negative emotion driving tendencies. Therefore, it may particularly useful for measuring risky driving temperament or propensity. Not surprisingly, we found that the DDDI risky driving subscale was more highly associated with the C-RDS and with Kinematic Risky Driving. C-RDS focuses exclusively on the prevalence of risky driving behaviors and appears to measure this construct reliably and with validity. Additional research is needed on these measures, but based on the findings reported here, we conclude the following:

- C-RDS is a reliable and valid instrument for prevalence surveys;

- DDDI Risky Driving Subscale may be a more useful measure of risky driving than the full DDDI. 


\section{ACKNOWLEDGMENTS}

This research was supported by the Intramural Research Program of the Eunice Kennedy Shriver National Institute of Child Health and Human Development, contract \# N01-HD-5-3405.

\section{REFERENCES}

Braitman, K.A., Kirley, B.B., McCartt, A.T., \& Chaudhary, N.K. (2008). Crashes of novice teenage drivers: Characteristics and contributing factors. Journal of Safety Research, 39, 4754.

Curry, A.E., Hafetz, J., Kallan, M.J., Winston, F.K., Durbin, D.R. Prevalence of teen driver errors leading to serious motor vehicle crashes. Accident Analysis \& Prevention, 43(6), 12851290.

Dula, C.S., \& Ballard, M.E. (2003). Development and evaluation of a measure of dangerous, aggressive, and negative emotional, and risky driving. Journal of Applied Social Psychology, 33(2), 263-282.

Hartos JL, Eitel P, Simons-Morton BG. Parenting Practices and Adolescent Risky Driving Behaviors: A Three-Month Prospective Study. Health Education \& Behavior, 29(2), 194206, 2002.

McKenna, F.P., Waylen, A.E., \& Burkes, M.E. (1998). Male and female drivers: how different are they? Berkshire, United Kingdom: The University of Reading, AA Foundation for Road Safety Research.

McKnight, J.A. \& McKnight, S.A. (2003). Young novice drivers: Careless or clueless. Accident Analysis \& Prevention, 35, 921-925.

Mayhew, D.R., Simpson, H.M., \& Pak, A. (2003). Changes in collision rates among novice drivers during the first months of driving. Accident Analysis \& Prevention, 35, 683-91.

McCartt, A.T., Shabanova, V.I., \& Leaf, W.A. (2003). Driving experience, crashes, and traffic citations of teenage beginning drivers. Accident Analysis \& Prevention, 35, 311-20.

Ouimet, M.C., Simons-Morton, B.G., Zador, P.L., Lerner, N.D., Freedman, M., Duncan, G.D., \& Wang, J. (2010). Using the U.S. National Household Travel Survey to estimate the impact of passenger characteristics on young drivers' relative risk of fatal crash involvement. Accident Analysis \& Prevention, 42(2), 689-94.

Richer, I., \& Bergeron, J. (2012). Differentiating risky and aggressive driving: Further support of the internal validity of the Dula Dangerous Driving Index. Accident Analysis \& Prevention, 45, 620-627.

Shope, J.T., \& Bingham, R.C. (2002). Drinking-Driving as a Component of Problem Driving and Problem Behavior in Young Adults. Journal of Studies on Alcohol, 63, 24-33.

Simons-Morton, B.G., Hartos, J., Leaf, W., \& Preusser, D. (2006). The Effects of the Checkpoints Program on Novice Teen Driving Limits and Outcomes. Accident Analysis and Prevention, 38, 907-912 
Simons-Morton, B.G., Ouimet, M.C., Zhang, Z., Lee, S.L., Klauer, S.E., Wang, J., Chen, R., Albert, P.E., \& Dingus, T.E. (2011). Crash and Risky Driving Involvement Among Novice Adolescent Drivers and Their Parents. American Journal of Public Health, 101 (12), 23622367.

Simons-Morton, B.G., Zhang, Z., Jackson, J.C., \& Albert, P.S. (2012). Do Elevated Gravitational-Force Events While Driving Predict Crashes and Near Crashes? American Journal of Epidemiology, 175(10), 1075-1079.

Willemsen, J., Dula, C.S., Declercq, F., \& Verhaeghe, P. (2008). The Dula Dangerous Driving Index: An investigator of reliability and validity across cultures. Accident Analysis \& Prevention, 40, 2, 798-806.

Williams, A.F. (2003). Teenage drivers: patterns of risk. Journal of Safety Research, 4(1), 5-15. 